\title{
Management of prostate cancer patients during COVID-19 pandemic
}

\author{
Can Obek $^{1} \cdot$ Tunkut Doganca $^{2} \cdot$ Omer Burak Argun ${ }^{1} \cdot$ Ali Riza Kural $^{1}$
}

Received: 5 May 2020 / Revised: 20 June 2020 / Accepted: 10 July 2020 / Published online: 20 July 2020

(c) The Author(s), under exclusive licence to Springer Nature Limited 2020

\begin{abstract}
Prostate cancer patients' management demands prioritization, adjustments, and a tailored approach during the unprecedented SARS-CoV-2 pandemic. Benefit of care from treatment must be carefully weighed against the potential of infection and morbidity from COVID-19. Furthermore, urologists need to be cognizant of their obligation for wise consumption of restricted healthcare resources and protection of the safety of their coworkers. Nonurgent in-person clinic visits should be postponed or conducted remotely via phone or teleconference. Prostate cancer screening, imaging, and biopsies may be suspended in general. Treatment may be safely deferred in low and intermediate risk patients. Surgery may be delayed in most high-risk patients and neoadjuvant ADT is generally not advocated prior to surgery. Initiation of long-term ADT coupled with EBRT subsequent to the pandemic may be favored as a feasible alternative in high-risk and very high-risk disease. In patients with $\mathrm{cN} 1$ disease, treatment within 6 weeks is advocated. Presurgery assessment should include testing for COVID-19 and preferably a chest imaging. In the presence of SARS-CoV-2 infection, surgery should be postponed whenever possible. All protective measurements suggested by national/international authorities must to be diligently followed during perioperative period. Strict precautions specific to laparoscopic/robotic surgery are required, considering the unproven but potential risk of aerosolization of SARS-CoV-2 virus and spillage with pneumoperitoneum. Regarding radiotherapy, shortest safe EBRT regimen should be favored and prophylactic whole pelvic RT and brachytherapy avoided. Chemotherapy should be avoided whenever possible.
\end{abstract}

\section{Introduction}

The global pandemic of the novel coronavirus SARS$\mathrm{CoV}-2$ is characterized by rapid human-to-human transmission. Initial reports from the epicenters of the outbreak have shown that the incidence of COVID-19 infection is higher in individuals with cancer [1]. Moreover, patients with a history of cancer or receipt of anticancer therapy within 14 days of presentation experience a higher incidence of severe events such as admission to intensive care unit (ICU), requirement for invasive ventilation, and death [2-4]. In a meta-analysis, the odds ratio of severe complications was 2.29 (95\% CI 1.00-5.23) [5]. Notably, recurrent hospital visits or admissions represent a risk for

Tunkut Doganca

tunkutdoganca@gmail.com

1 Department of Urology, Acibadem Mehmet Ali Aydinlar University Medical Faculty, Istanbul, Turkey

2 Department of Urology, Acibadem Taksim Hospital, Istanbul, Turkey acquiring infection with SARS-CoV-2. Thus, physicians and cancer patients alike are seriously challenged with the dilemma of weighing the benefit of routine cancer care against the potential morbidity and mortality of COVID19 infection throughout the unprecedented SARS-CoV-2 pandemic. Another very important aspect is the obligation for judicious utilization of limited healthcare resources [6]. Last but not least, serious consideration must be given to mitigating COVID-19 exposure to healthcare personnel.

Governing healthcare authorities have published or released recommendations on the appropriate courses of action to adapt urology practices to the current crisis. We have made a thorough PubMed and web-based search on every significant paper/statement/guideline/recommendation published and/or released related to prostate cancer (PC) management during the COVID-19 global outbreak. Recommendations covering all aspects of the disease, such as diagnosis, staging, management based on stage and risk stratification, surgery (open and laparoscopic/robotic), systemic treatment, and radiotherapy, were incorporated. To the best of our knowledge, this represents the first thorough review and synopsis of currently available 
recommendations on the optimal management of $\mathrm{PC}$ patients during SARS-CoV-2 pandemic and has been revised on May 19, 2020. Of note, the recommendations released so far address the challenges in the acute phase of the pandemic (with one exception [7]) and will be subject to modifications with new scientific data and evolving status of the pandemic in days/months to come. They should not be perceived as rigid guidelines established from high level of evidence, but rather as reasonable perspectives on risk/ benefit ratio in specific clinical scenarios. A particular advice may not apply to all countries, regions, or institutions, as a number of variables, including the stage of the pandemic, local healthcare capacity, risk of infection to a certain individual, stage and grade of cancer, presence of symptoms, comorbidities, age, and details of the treatment, must be weighed in the equation [8].

\section{Resource allocation}

In extraordinary circumstances of severely limited capacity, ethical principles emphasize treating patients fairly, equitably, and safely, according to need [3, 9]. The American Society of Clinical Oncology (ASCO) has published recommendations on ethics and resource scarcity [10]. Decisions regarding allocation of scarce resources should be separated from bedside decision-making, and the physician taking care of the patient should not make resource allocation decisions [10]. These decisions should be made by a triage committee. Physicians involved in cancer care may support these decisions by providing accurate and up-todate evidence on disease prognosis and treatment options for an individual patient [10]. A cancer diagnosis should not keep an individual from having a fair chance to access scarce resources. PC patients and their families would be expected to experience heightened vulnerability and psychological stress during the outbreak. Furthermore, some will be affected by economic instability and face occupational insecurity and subsequent discontinuation of access to health coverage benefits [11]. Physicians in charge should communicate allocation plan and decisions to their patients and their loved ones personally, with compassion and honesty [10]. Patients should ideally be involved in the decision-making process [11].

\section{Tumor boards and clinical trials}

Multidisciplinary oncology meetings should continue and be virtualized over the phone or video conferences [12-15]. Recruitment to clinical trials would require careful consideration; pausing recruitment to trials and diverting available resources to fight the pandemic may be appropriate [16].

\section{Screening}

National Comprehensive Cancer Network (NCCN), European Association of Urology (EAU), and the Canadian Framework advise against routine PC screening, including prostate specific antigen (PSA) testing and digital rectal examination (DRE), for all asymptomatic individuals until the pandemic subsides $[11,14,17]$. This is based on the fact that the risks of a delay in diagnosis of up to 6-12 months would be marginal for most PC [17].

\section{Biopsy}

For individuals already evaluated and harbor an elevated PSA and/or abnormal DRE, NCCN and the Canadian Framework advise to suspend further testing and biopsy until after the pandemic [11, 17]. In exceptional circumstances when prostate biopsy is deemed essential for diagnosis of a potentially lethal PC, an urgent biopsy may be considered $[11,17]$. EAU advocates a more staged approach to biopsy. When PSA is $<10 \mathrm{ng} / \mathrm{ml}$ and DRE feels benign, further evaluation may be postponed until pandemic subsides [14]. However, when PSA is $>10 \mathrm{ng} / \mathrm{ml}$ or DRE is abnormal, biopsy may be performed in 3-4 months [14]. Similar to EAU, American Urological Association (AUA) recommends a risk-based approach [18]. For individuals with a PSA $>20$, PSA DT $<6$ months, DRE suggestive of T3 disease, AUA suggests biopsy delay up to 3 months and a transperineal approach to avoid fecal exposure [18]. Biopsy may be delayed for 3-6 months in all others. If DRE suggests locally advanced disease or the patient is symptomatic, then biopsy would need to be performed within 6 weeks [14]. For individuals presenting with symptoms of metastasis, EAU advocates imaging for staging in $<6$ weeks, and if metastasis is confirmed, commencing with androgen deprivation therapy (ADT) while postponing biopsy [14]. Full staging evaluation, including laboratory testing and imaging, is recommended in advanced PC (clinical nodal involvement, metastatic disease) [11].

\section{Clinic visits}

All nonurgent in-person clinic visits should be canceled or postponed and conducted remotely as telehealth communication, unless patient suffers from active symptoms, requires wound care, or presents with newly diagnosed advanced PC [11, 12, 17, 19]. Initial PSA after definitive local treatment could be suspended; alternatively, virtual visits with discussions of PSA and testosterone results could be considered [17]. Genetic counseling may be performed virtually [17]. During monitorization of disease, scheduled imaging may be deferred if PSA is declining and the patient 
Table 1 Summary of management recommendations for prostate cancer during acute phase of Covid-19 pandemic.

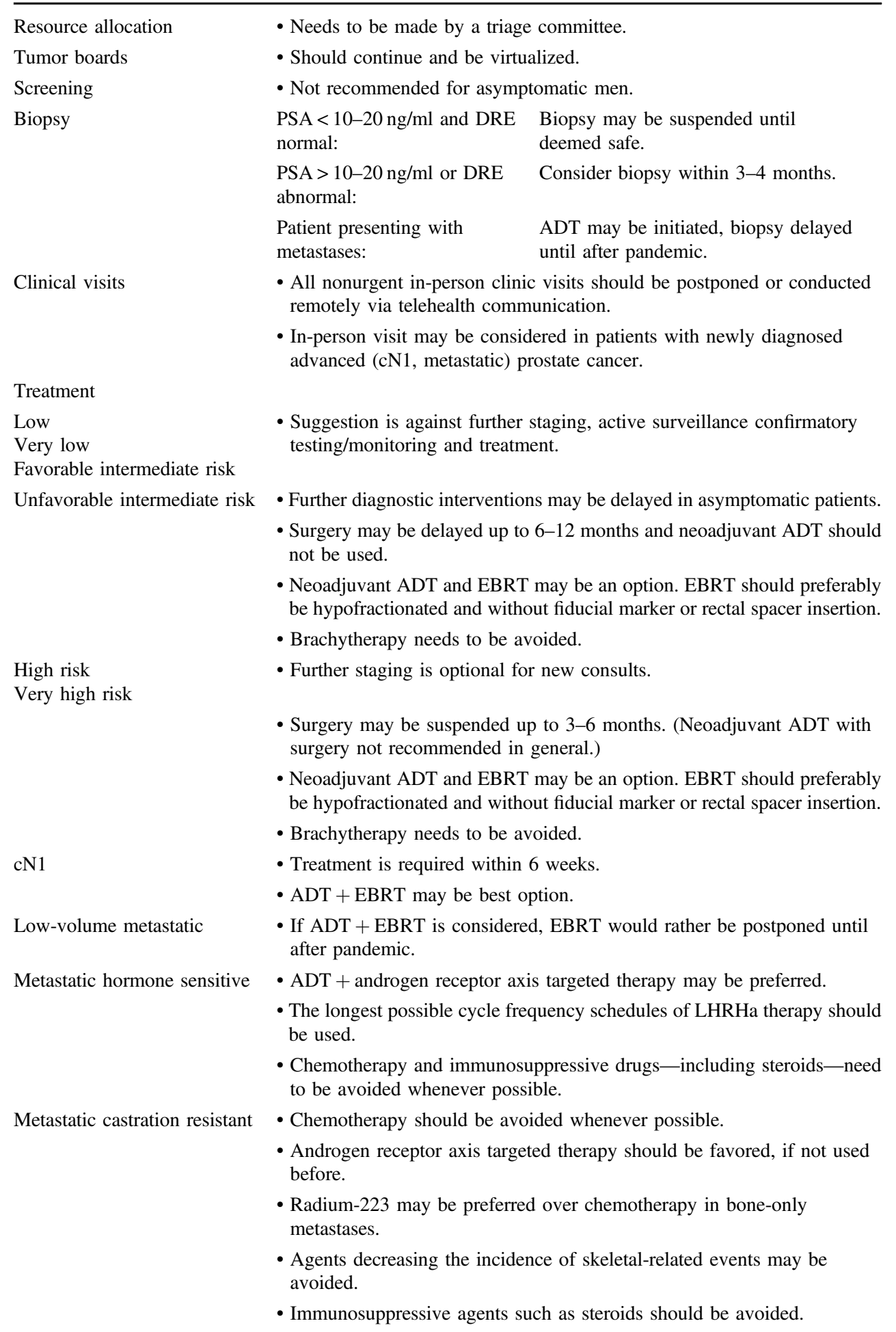

National Comprehensive Cancer Network risk stratification is used.

$P S A$ prostate specific antigen, DRE digital rectal examination, $A D T$ androgen deprivation therapy, EBRT external beam radiotherapy, $L H R H a$ luteinizing hormone releasing hormone agonist. is asymptomatic, until risk of COVID-19 has resolved [17]. For patients with NCCN asymptomatic unfavorable intermediate risk (UIR), high-risk (HR), and very HR (VHR) $\mathrm{PC}$, further staging may be delayed, until restrictions are lifted [17]. In the presence of persistently elevated PSA or PSA relapse after treatment with curative intent, imaging could be postponed [14]. Suggestions regarding screening and biopsy are summarized in Table 1. 


\section{Treatment by stage}

\section{Local, locally advanced, and regional disease}

PC is a slowly progressive disease in general and NCCN cautions against overestimating the benefit of routine localized PC care [13, 20]. Consequently, minimal harm would be expected with delays in treatment, exclusively as weighed against the risk of mortality from SARS-CoV-2 infection $[14,17]$.

Patients with very low, low, and favorable intermediate risk (FIR) disease are recommended not to undergo further staging, active surveillance, confirmatory testing/monitoring, and treatment, until deemed safe [11, 14, 17]. The length of delay until adverse outcomes occur is unknown; however, based on retrospective data, 6-12 months are likely reasonable $[11,14]$.

For patients with intermediate risk disease, if active surveillance in Gleason $3+4$ disease is deemed as an option, it may be deferred for 3-4 months [14]. If surgery is the preferred option, delaying radical prostatectomy (RP) until after the pandemic is advocated [11, 14]. Neoadjuvant ADT should not be used to bridge the COVID-19 related delay to surgery $[11,14]$. When consideration is given to radiotherapy, suggestion is to use external beam radiotherapy (EBRT) with hypofractionation and avoidance of invasive procedures such as fiducial insertion and/or rectal spacers $[11,14,21]$. EAU suggests initiating with neoadjuvant ADT that might be prolonged for up to 6 months. While Canadian Framework concurs with neoadjuvant hormonal therapy with EBRT for UIR disease, they advise against using neoadjuvant ADT in patients with FIR disease $[11,14]$. Recommendations in general are against performing brachytherapy during the pandemic due to its invasive nature and potential inherent infectious complications [14, 21]. For individuals with asymptomatic UIR disease, NCCN advocates delaying further work-up and treatment, but Canadian Framework recommends proceeding with diagnostic interventions in cases with new consult UIR, based on the fact that finding of metastases would alter management $[11,17]$.

HR PC remains the most challenging to manage. NCCN indicates that further staging and radical treatment may be delayed for patients with HR and VHR PC, until deemed safe $[15,17]$. EAU, likewise, recommends postponing surgery until after the pandemic or if the patient is anxious, consideration given to ADT + EBRT [14]. Data from Johns Hopkins suggests delaying surgery in UIR and HR patients upward of 6 months would not adversely impact outcome $[17,22]$. In a recent article, Ginsburg et al. performed a retrospective review of patients with intermediate and HR PC in the National Cancer Database to address the implications of delays due to the viral outbreak. They concluded that a delay of RP up to 12 months was not associated with early adverse oncological outcomes such as upgrading on $\mathrm{RP}$, node-positive disease, and postsurgery secondary treatments. These results may provide reassurance to patients and urologists [23]. However, biochemical recurrence rates may be higher in HR men who delay definitive treatment $[15,24,25]$. Canadian Framework has a somewhat more restrained approach. Authors caution that a delay of more than 3 months may jeopardize oncological outcomes $[11,26]$. Although authors recognize that neoadjuvant ADT prior to surgery is normally not recommended outside of clinical trials, they state that upfront ADT may be an option in patients with UIR, HR, and VHR disease during COVID-19 crisis, if prolonged surgical delays are expected [11].

In patients with locally advanced disease including $\mathrm{cN} 1$, treatment should be initiated within 6 weeks [14]. Canadian Framework advocates ADT and EBRT, as current best practice [11]. Although abiraterone has shown benefit in these patients, it would rather be postponed during the pandemic [11]. Concerning surgery, EAU encourages against using neoadjuvant ADT to postpone RP and considering long-term ADT + EBRT as an alternative to surgery [14]. For men with low-volume metastatic disease in whom ADT + prostate EBRT is considered, EBRT should be postponed, until the pandemic is no longer a major threat [14]. For symptomatic patients (e.g., obstruction, bleeding), conservative measures should be prioritized (e.g., medical therapy, ADT, clean intermittent catheterization) [17].

When treatment is deemed crucial for persistently elevated PSA after surgery, recommendation would be to start ADT and postpone further work-up and potential EBRT for later [14]. Salvage EBRT should be favored rather than adjuvant radiation therapy (RT) $[11,14]$. For PSA relapse after EBRT, if salvage is needed, ADT would be appropriate initially, when PSA doubling time is $<12$ months [14]. Immediate treatment with decompressive surgery or upfront EBRT plus systemic therapy would be mandatory for patients presenting with impending spinal cord compression [14].

A brief synopsis of treatment recommendations is presented in Table 1.

\section{Surgery}

Urologists should support the healthcare system by reducing the demand for ventilators, personal protective equipment (PPE), and various other hospital and human resources by meticulous prioritization and minimization of surgeries without compromising patient outcomes in the context of medical and logistical considerations [15, 27]. The American College of Surgeons (ACS) and the American Society 
of Anesthesiologists and ASCO advocate surgical decisions to be made by a leadership team represented by surgery, anesthesiology, and nursing departments [10, 15, 27]. Consent covering the risk of SARS-CoV-2 virus exposure and consequences should be obtained. Infection with virus should be sought in any patient requiring surgery by history, testing, and preferably a recent chest imaging [14, 19, 28]. Real-time reverse transcription polymerase chain reaction (RT-PCR) assays detecting viral RNA have been most widely used to date. Since April 1, 2020, the Food and Drug Administration has approved rapid testing based on IgM and IgG antibodies [29]. Test result may be available within $20 \mathrm{~min}$ and the test has been currently approved as an adjunct to PCR testing with a sensitivity of 93.8\% [30]. Although there is no worldwide approval for the antibody test, it may be helpful before surgery in increasing sensitivity and determine asymptomatic carriers [30].

In case of COVID-19 test positivity, the procedure should be postponed, unless extremely emergent. In Wuhan experience, patients undergoing surgery during the incubation period experienced postoperative viral pneumonia in $100 \%$, required admission to ICUs in $44 \%$, and a mortality of $20.5 \%$ [31]. Nonetheless, urologists practicing in hospitals treating COVID-19 patients may face situations when they have to perform urgent procedures on infected individuals. Under these circumstances, procedures should be performed in dedicated operating rooms (OR) complying with the hospital recommendation for OR staff protection [14, 32]. Recently, Coccolini et al. have published on the presence of viral parts in peritoneal fluid by RT-PCR [33]. Although this is not proof of live and infectious virus in the peritoneal fluid, it is somewhat concerning regarding peroperative contamination. Surgeons and personnel not involved with intubation should remain outside the OR, until anesthesia induction and intubation are completed [14, 27, 34]. Intubation and extubation should take place within a negative pressure room, whenever possible [7, 35]. Surgical equipment used during procedures with confirmed or suspected COVID-19-positive patients should be cleaned separately [14, 19]. Using discrete spaces in ICU and patient care areas should be considered [27].

Even in the presence of a negative COVID-19 PCR test result, all recommendations to mitigate SARS-CoV-2 transmission must be diligently followed, due to the likelihood of false-negative test results [30, 32]. Only essential staff should participate in surgical case without exchange of room personnel. Entire OR staff should use PPE as indorsed by national/international authorities [19, 32]. Energy-based surgical instruments have the potential to produce particles sufficiently small $(<5 \mu \mathrm{M})$ for aerosolization in surgical smoke [36]. Therefore, efforts should include setting electro-surgery units to the lowest possible settings for the desired effect and minimizing use of monopolar electro-surgery, ultrasonic dissectors, and bipolar devices [19, 37-39].

There has been controversy regarding the safety of performing laparoscopy/robot-assisted surgery during COVID19 pandemic. The particle concentration of smoke in laparoscopic surgery may be significantly higher than open surgery [32]. Previous research has shown that laparoscopy can lead to aerosolization of other blood borne viruses and evacuation of pneumoperitoneum may results in spillage of aerosolized blood particles [28, 40-42]. Van Doremalen et al. have verified in vitro evidence that SARS-CoV-2 is similar to SARS-CoV-1 with plausible transmission of the virus via aerosol [43]. Thus, concern has been raised that $\mathrm{CO}_{2}$ could theoretically contain COVID-19 particles and aerosolization would expose OR personnel to the infectious virus, in the presence of uncontrolled release of gas into the surgical field [19, 28, 30]. Nevertheless, it should be underlined that there is yet no evidence to indicate that SARS-CoV-2 virus is present in $\mathrm{CO}_{2}$ aeroseol [36, 37]. Besides, it remains unknown whether inhalation of surgical smoke containing aerosolized viral particles leads to clinical infection. Although a recent publication has raised concerns about potential aerosol transmission of SARS-CoV-2 and offered recommendations, it remains merely level $\mathrm{V}$ evidence [44]. ACS's initial suggestion for consideration given to "avoiding laparoscopy" was later updated to "there are insufficient data to recommend for/against open versus laparoscopy approach" [27]. Likewise, Royal College of Surgeons' Updated Intercollegiate General Surgery Guidance on COVID-19 initial statement of "laparoscopy should generally not be used during the pandemic" was later changed to "consider laparoscopy only in selected individual cases, where clinical benefit to the patient substantially exceeds the risk of viral transmission to surgical and theater teams" [27, 28]. Most recent Society of Urologic Robotic Surgeons (SURS) recommendation is to limit minimally invasive surgery (MIS) to planned urgent or emergency procedures throughout the outbreak [30]. Porter et al. revealed that there have been no reports of COVID-19 related fatality of healthcare workers directly attributed MIS [30]. Contradictory opinions point out to potential advantages of laparoscopic surgery during the pandemic, given all necessary precautions during perioperative period are fulfilled. Society of American Gastrointestinal and Endoscopic Surgeons (SAGES) emphasizes proven benefits of MIS on reduced hospitalization and complications, which might be advantageous during a period of restricted resources [19]. SAGES also raises concerns that filtration of aerosolized particles may be even more challenging during open surgery [19].

Nonetheless, erring on the side of safety would warrant treating SARS-CoV-2 as exhibiting infectious aerosolization properties and specific precautions need to be taken for 
Table 2 Recommendations for surgery during Covid-19 pandemic.

- Consent covering the risk of SARS-CoV-2 virus exposure and consequences should be obtained.

- Infection with virus should be sought in any patient requiring surgery by history, testing and preferably a recent chest imaging.

- In case of COVID-19 test positivity, the procedure should be postponed, unless extremely emergent.

- When surgery must be performed in a patient with known or suspected COVID-19 infection, procedures should be performed in dedicated ORs complying with the hospital recommendation for OR staff protection. Intubation and extubation should take place within a negative pressure room.

- In the presence of a negative COVID-19 PCR test result, all recommendations to mitigate SARS-CoV-2 transmission must be diligently followed.

- Electro-surgery units should be set at the lowest possible settings.

- There are precautions specific to laparoscopy/robotic surgery that need to be fulfilled.

- There is yet no evidence to indicate that SARS-CoV-2 virus is present in $\mathrm{CO}_{2}$ aerosol; however, erring on the side of safety would warrant treating SARS-CoV-2 as exhibiting infectious aerosolization.

- Efforts should be made to limit cautery plume creation and uncontrolled $\mathrm{CO}_{2}$ release into the OR.

- Insufflation pressure should be kept to the lowest acceptable level offering a safe operating space and maintain visibility.

- For robot-assisted laparoscopy, the use of intelligent integrated insufflation systems is recommended.

- Laparoscopic suction devices should ideally be connected to a filtered device with an ULPA or HEPA filter.

- Prior to specimen extraction, closure, trocar removal, or conversion to open, all pneumoperitoneum should be safely evacuated via a filtration system.

$O R$ operating room, ULPA ultralow particulate air, HEPA highefficiency particulate air.

laparoscopy/robotic surgery [30]. MIS should be performed by experienced surgeons and surgical training avoided $[30,32,37]$. No visitors should be allowed in the OR and personnel exchange kept to a minimum [27, 30, 32, 37]. PPE, including N-95 masks, should be made available to healthcare staff [30]. Efforts should be made to limit cautery plume creation and uncontrolled $\mathrm{CO}_{2}$ release into the $\mathrm{OR}$ [30]. Nasogastric tube placement is an aerosol generating procedure requiring full PPE and preferably should be carried out in a specified location [28]. Port incisions should be of minimum length to allow for passage of ports, but not allow for leakage. An $8 \mathrm{~mm}$ instrument should not be introduced through a $12 \mathrm{~mm}$ daVinci trocar without a reducer and a $5 \mathrm{~mm}$ instrument should not be introduced in a $12 \mathrm{~mm}$ trocar [19, 37]. If ports develop a leak, the seals/ ports should be changed immediately [45]. Insufflation pressure should be kept to the lowest acceptable level offering a safe operating space and maintain visibility $[19,30,37]$. Smoke evacuation systems with active filtering mode should be provided [19, 37]. For robot-assisted laparoscopy, the use of intelligent integrated insufflation systems is recommended $[32,37]$. One of the available systems that allows for a closed circulation of $\mathrm{CO}_{2}$ along with ultralow particulate air (ULPA) filtration is the smoke evacuation mode of Airseal System (Conmed, USA; Surgiquest, UK) [30]. Prior to specimen extraction, closure, trocar removal, or conversion to open, all pneumoperitoneum should be safely evacuated via a filtration system $[30,37]$. Laparoscopic suction devices should ideally be connected to a filtered device with an ULPA, or highefficiency particulate air filter and not to an in-room canister connected to wall suction [30]. For very detailed information on necessary precautions and technical requirements to mitigate viral contamination during laparoscopic and robotic surgery, please refer to the SURS recommendations [30]. Table 2 briefly summarizes surgical precautions.

While in hospital during recovery from surgery, cancer patients should be considered immunocompromised and at high risk of severe consequences with COVID-19 infection. Appropriate precautions and early discharge is encouraged [12]. Visitors to COVID-19 patients need to be limited or preferably not allowed.

\section{Radiation therapy}

Radiation oncologists from the United States and United Kingdom have published their recommendations on safe management of PC during the outbreak [21]. They developed and published the Remote visits, and Avoidance, Deferment, and Shortening of radiotherapy Framework.

RT may be preferred over surgery in HR patients during the pandemic. This would require a shared decision with the patient. Neoadjuvant ADT should be considered in asymptomatic UIR, HR, cN1, oligometastatic, and low-volume M1 patients planning to receive definitive EBRT [21]. This may safely be given for up to 6 months [17]. In attempts to decrease the number of hospital visits, shortest safe EBRT regimen should be favored, which may consist of 5-7 fractions of SBRT/ultrahypofractionation for localized IR and HR PC [17, 21]. Prophylactic whole pelvic RT should generally be avoided, due to the increased risk of grade IV lymphopenia (unpublished data from RTOG 94139 [46]). As previously mentioned, invasive procedures such as fiducial insertion and/or rectal spacers for EBRT and brachytherapy should be avoided given their reliance on anesthesia staff and PPE, risk of complications including severe infection and hospitalization [11, 21]. Regarding palliative radiotherapy, alternative options such as oral analgesics should be preferred during the pandemic [21]. For more detailed information on technical details of radiotherapy, please refer to the publication by Zaorsky et al. [21]. 


\section{Systemic treatment}

Man with advanced PC is generally older, frail, and has multiple comorbidities. The risk/benefit ratio of palliative and neoadjuvant/adjuvant treatments needs to be meticulously assessed. Minimizing potential exposure to COVID19 by reducing visits to clinic, particularly for intravenous or inpatient therapy, is advised. This is particularly important during the early phase of the pandemic, when incidence is rising exponentially and the imminent pressure on healthcare resources remains unknown [8, 16, 21, 47, 48]. Regimens with a clear survival advantage should be prioritized with curative treatments remaining mandatory and others requiring consideration of the risk/benefit ratio [16]. Treatments that have only shown a palliative effect for patients who are symptomatic require careful discussion.

For patients with nonmetastatic disease and a PSA doubling time of $>9$ months, initiating ADT may be suspended [16]. Systemic treatment should be offered to patients with hormone sensitive M1 disease; standard of care would be ADT + androgen receptor axis targeted therapy (ARAT) $[9,11,14]$. Docetaxel should be avoided as much as possible based on the risk of neutropenia and frequent hospital visits during the pandemic $[9,11,14,16]$. The initiation of ARATs may be delayed up to 6 months in this climate [9]. Utilization of abiraterone acetate with $5 \mathrm{mg}$ prednisone daily might be reconsidered with respect to avoidance of steroid [14]. Once ADT is instigated, remote telemonitorization for PSA, testosterone, and other laboratory results would be appropriate [17]. For patients on luteinizing hormone releasing hormone analogs, the longest possible cycle frequency schedules of therapy should be used, e.g., q 3-6 months instead of monthly injections $[11,12,17]$.

EAU considers treatment for metastatic castration resistant (mCRPC) as high priority. Thus treatment with lifeprolonging agents should be initiated in $<6$ weeks [14]. Chemotherapy should be avoided as much as possible, and when absolutely needed, docetaxel $75 \mathrm{mg} / \mathrm{m}^{2}$ should be given 3-weekly with systematic G-CSF to avoid more frequent visits or with $50 \mathrm{mg} / \mathrm{m}^{2}$ every 2 weeks $[12,14,17]$. Cabazitaxel $20 \mathrm{mg} / \mathrm{m}^{2}$ with systematic G-CSF should be given if indicated and no other treatment option is available [14]. Canadian recommendation for mCRPC would be in favor of ARATs, if not used before [9, 11]. If ARATs have been used previously, such that chemotherapy would be next-line recommendation, a thorough discussion and shared decision would be in order between the patient and the physician [9]. In patients with bone-only mCRPC, radium-223 may be preferred over chemotherapy, based on availability [9, 11]. Agents decreasing the incidence of skeletal-related events may be avoided or intervals extended, if administration of therapy involves hospital visits
[11, 12, 14]. Immunosuppressive agents such as steroids should be avoided or reduced for antiemesis and prolonged steroid treatment for PC should require reassessment [9, 14]. Nonmyelosuppressive regimens must be preferred, when alternatives exist [17]. Sipuleucel-T treatment may be deferred [14, 17].

A recent retrospective population study from Italy has suggested of a partial protective effect of ADT from SARSCoV-2 infection [49]. It is based on the rationale that SARS-CoV-2 uses TMPRSS2, a member of the family of type II transmembrane serine proteases, for fusion of viral and cellular membranes. TMPRSS2 is highly expressed in PC and its transcription is regulated by the androgen receptor. The authors showed that PC patients receiving ADT had a significantly lower risk of COVID-19 infection (OR 4.05; 95\% CI 1.55-10.59) [49]. More evidence on this topic would be essential to draw any meaningful conclusions.

\section{Roadmap to resuming elective surgery after COVID-19 pandemic}

US governing health authorities have released a joint statement on a roadmap for resuming elective surgery after COVID-19 pandemic [7]. There are three main criteria that must be met. There should be a sustained reduction in the rate of new COVID-19 cases in the relevant geographic area for at least 14 days. Any resumption should be authorized by the appropriate municipal, country, and state health authorities. The facility should have appropriate number of ICU and non-ICU beds, PPE, ventilators, and trained staff to treat all nonelective patients without resorting to a crisis standard of care [7].

\section{Conclusions}

We are facing a clinical conundrum without a modern precedent. Most routine activities regarding screening, diagnosis, and treatment of PC may be safely suspended during the SARS-CoV-2 pandemic. In-person visits should be replaced by virtual visits, whenever possible. Most surgeries may be deferred, until after the pandemic. HR patients may be counseled on neoadjuvant ADT + EBRT or delaying surgery. Precautions specific to laparoscopic/ robotic surgery need to be fulfilled, considering the unproven but potential risk of aerosolization of SARS-CoV2 virus and spillage with pneumoperitoneum. The shortest safe EBRT regimen should be favored and prophylactic whole pelvic RT should generally be avoided. Systemic therapy regimens with a clear survival advantage should be prioritized, with curative treatments remaining mandatory 
and others requiring consideration of the risk/benefit ratio. Nonmyelosuppressive regimens should be favored, when alternatives exist. Prioritization strategies and recommendations may be subject to change and thus have to be reevaluated frequently based on the trajectory of COVID-19, international and national health authority recommendations and resources available at institutions.

\section{Compliance with ethical standards}

Conflict of interest The authors declare that they have no conflict of interest.

Publisher's note Springer Nature remains neutral with regard to jurisdictional claims in published maps and institutional affiliations.

\section{References}

1. Yu J, Ouyang W, Chua MLK, Xie C. SARS-CoV-2 rransmission in patients with cancer at a tertiary care hospital in Wuhan, China. JAMA Oncol. 2020;6:1108-10.

2. Ng OT, Marimuthu K, Chia PY, Koh V, Chiew CJ, De Wang L, et al. SARS-CoV-2 infection among travelers returning from Wuhan, China. N Engl J Med. 2020;382:1476-8.

3. Persad G, Wertheimer A, Emanuel EJ. Principles for allocation of scarce medical interventions. Lancet. 2009;373:423-31.

4. Zhang L, Zhu F, Xie L, Wang C, Wang J, Chen R, et al. Clinical characteristics of COVID-19-infected cancer patients: a retrospective case study in three hospitals within Wuhan, China. Ann Oncol. 2020;31:894-901.

5. Wang B, Li R, Lu Z, Huang Y. Does comorbidity increase the risk of patients with COVID-19: evidence from meta-analysis. Aging 2020;12:6049-57.

6. Siegel RL, Miller KD, Jemal A. Cancer statistics, 2019. CA: Cancer J Clin 2019;69:7-34.

7. American College of Surgeons, American Society of Anesthesiologists, Association of periOperative Registered Nurses, American Hospital Association. Joint statement: roadmap for resuming elective surgery after COVID-19 pandemic. American College of Surgeons, American Society of Anesthesiologists, Association of periOperative Registered Nurses, American Hospital Association; 2020. https://www.aorn.org/guidelines/aornsupport/roadmap-for-resuming-elective-surgery-after-covid-19.

8. Anderson RM, Heesterbeek H, Klinkenberg D, Hollingsworth TD. How will country-based mitigation measures influence the course of the COVID-19 epidemic? Lancet. 2020;395:931-4.

9. Lalani AA, Chi KN, Heng DYC, Kollmannsberger CK, Sridhar SS, Blais N, et al. Prioritizing systemic therapies for genitourinary malignancies: Canadian recommendations during the COVID-19 pandemic. Can Urol Assoc J. 2020;14:E154-E8.

10. Marron JM, Joffe S, Jagsi R, Spence RA, Hlubocky FJ. Ethics and resource scarcity: ASCO recommendations for the oncology community during the COVID-19 pandemic. J Clin. Oncol. 2020;38:2201-5.

11. Kokorovic A, So AI, Hotte SJ, Black PC, Danielson B, Emmenegger $U$, et al. A Canadian framework for managing prostate cancer during the COVID-19 pandemic: recommendations from the Canadian Urologic Oncology Group and the Canadian Urological Association. Can Urol Assoc J. 2020;14:163-8.

12. British Columbia Cancer Agency. Provincial cancer clinical management guidelines in pandemic situation (COVID-19). British
Columbia Cancer Agency, 2020. http://www.bccancer.bc.ca/hea lth-professionals-site/Documents/BCCancerPandemicClinicalMa nagementGuidelines.pdf.

13. Herget KA, Patel DP, Hanson HA, Sweeney C, Lowrance WT. Recent decline in prostate cancer incidence in the United States, by age, stage, and Gleason score. Cancer Med. 2016;5:136-41.

14. Ribal MJ, Cornford P, Briganti A, Knoll T, Gravas S, Babjuk M, et al. EAU Guidelines Office Rapid Reaction Group: an organisation-wide collaborative effort to adapt the EAU guidelines recommendations to the COVID-19 era. Eur Urol. 2020;78:21-8. https://www.uroweb.org/wp-content/uploads/EAU-GuidelinesOffice-Rapid-Reaction-Group-An-organisation-wide-collabora tive-effort-to-adapt-the-EAU-guidelines-recommendations-to-theCOVID-19-era.pdf.

15. Stensland KD, Morgan TM, Moinzadeh A, Lee CT, Briganti A, Catto JWF, et al. Considerations in the triage of urologic surgeries during the COVID-19 pandemic. Eur Urol. 2020. https://www. europeanurology.com/covid-19-resource.

16. Gillessen S, Powles T. Advice regarding systemic therapy in patients with urological cancers during the COVID-19 pandemic. Eur Urol. 2020;77:667-8. https://www.europeanurology.com/ article/S0302-2838(20)30201-3.

17. Mohler J, Srinivas S, Antonarakis ES, Armstrong AJ, Bekelman JE, Cheng $\mathrm{H}$, et al. NCCN guidelines version 1.2020. Prostate Cancer. 2020. https://www.nccn.org/professionals/physician_gls/ pdf/prostate.pdf.

18. Katz EG, Stensland KS, Mandeville JA, MacLachlan LS, Moinzadeh A, Sorcini A, et al. Triaging office-based urology procedures during the COVID-19 pandemic. J Urol. 2020. https://doi. org/10.1097/JU.0000000000001034.

19. SAGES. SAGES and EAES recommendations regarding surgical response to COVID-19 crisis. SAGES; 2020. https://www.sages. org/recommendations-surgical-response-covid-19/.

20. Kohler BA, Sherman RL, Howlader N, Jemal A, Ryerson AB, Henry KA, et al. Annual report to the nation on the status of cancer, 1975-2011, featuring incidence of breast cancer subtypes by race/ethnicity, poverty, and state. J Natl Cancer Inst. 2015;107: djv048.

21. Zaorsky NG, Yu JB, McBride SM, Dess RT, Jackson WC, Mahal $\mathrm{BA}$, et al. Prostate cancer radiotherapy recommendations in response to COVID-19. Adv Radiat Oncol. 2020. https://doi.org/ 10.1016/j.adro.2020.03.010. [Online ahead of print].

22. Siegel RL, Miller KD, Jemal A. Cancer atatistics, 2017. CA: Cancer J Clin. 2017;67:7-30.

23. Ginsburg KB, Curtis GL, Timar RE, George AK, Cher ML. Delayed radical prostatectomy is not associated with adverse oncological outcomes: implications for men experiencing surgical delay due to the COVID-19 pandemic. J Urol. 2020. https://doi. org/10.1097/JU.0000000000001089.

24. Fossati N, Rossi MS, Cucchiara V, Gandaglia G, Dell'Oglio P, Moschini M, et al. Evaluating the effect of time from prostate cancer diagnosis to radical prostatectomy on cancer control: can surgery be postponed safely? Urologic Oncol. 2017;35:150.e9-15.

25. Loeb S, Folkvaljon Y, Robinson D, Makarov DV, Bratt O, Garmo $\mathrm{H}$, et al. Immediate versus delayed prostatectomy: nationwide population-based study (.). Scand J Urol. 2016;50:246-54.

26. Reichard CA, Nyame YA, Sundi D, Tosoian J, Wilkins L, Alam R, et al. Does time from diagnosis to treatment of high- or very-highrisk prostate cancer affect outcome? BJU Int. 2019;124:282-9.

27. The American College of Surgeons. ACS guidelines for triage and management of elective cancer surgery cases during the acute and recovery phases of coronavirus disease 2019 (COVID-19) pandemic. The American College of Surgeons; 2020. https://www.fa cs.org/-//media/files/covid19/acs_triage_and_management_ elective_cancer_surgery_during_acute_and_recovery_phases. ashx. 
28. Intercollegiate general surgery guidance on COVID-19 update. Royal College of Surgeons of Edinburgh, 2020. https://www. rcsed.ac.uk/news-public-affairs/news/2020/march/intercollegiategeneral-surgery-guidance-on-covid-19-update.

29. Food and Drug Administration. Coronavirus (COVID-19) update: daily roundup April 2, 2020. Food and Drug Administration; 2020. https://www.fda.gov/news-events/press-announcements/ coronavirus-covid-19-update-daily-roundup-april-2-2020.

30. Porter J, Blau E, Gharagozloo F, Martino M, Cerfolio R, Duvvuri U, et al. Society of Robotic Surgery Review: recommendations regarding the risk of COVID-19 transmission during minimally invasive surgery. BJU Int. 2020. https://doi.org/10.1111/bju. 15105. [Online ahead of print].

31. Lei S, Jiang F, Su W, Chen C, Chen J, Mei W, et al. Clinical characteristics and outcomes of patients undergoing surgeries during the incubation period of COVID-19 infection. EClinicalMedicine. 2020;21:100331. https://doi.org/10.1016/j.eclinm. 2020.100331. [Online ahead of print].

32. Mottrie A, Puliatti, S, Mazzone, E. ERUS-EAU Robotic Urology Section ERUS (EAU Robotic Urology Section) guidelines during COVID-19 emergency. ERUS-EAU Robotic Urology Section. https://www.uroweb.org/wp-content/uploads/ERUS-guidelines-forCOVID-def.pdf.

33. Coccolini F, Tartaglia D, Puglisi A, Giordano C, Pistello M, Lodato M, et al. SARS-CoV-2 is present in peritoneal fluid in COVID-19 patients. Ann Surg. 2020. Article In press. Avaliable at https://www.journals.lww.com/annalsofsurgery/Documents/ SARS-CoV-2\%20is\%20present $\% 20$ in $\% 20$ peritoneal $\% 20$ fluid $\%$ 20in\%20COVID-19\%20patients.pdf.

34. Chow TT, Yang XY. Ventilation performance in operating theatres against airborne infection: review of research activities and practical guidance. J Hosp Infect. 2004;56:85-92.

35. National Health Service. National guidance. National Health Service. https://www.icmanaesthesiacovid-19.org/national-guidance, 2020.

36. Mowbray N, Ansell J, Warren N, Wall P, Torkington J. Is surgical smoke harmful to theater staff? A systematic review. Surg Endosc. 2013;27:3100-7.

37. EAU general recommendations for surgical procedures. https://www.uroweb.org/wp-content/uploads/Gen-Rec-Surg-ProcApril-14.pdf. European Association of Urology, 2020.

38. Parsa RS, Dirig NF, Eck IN, Payne III WK. Surgical smoke and the orthopedic implications. Internet J Orthop Surg. 2016;24:1.
39. Wisniewski PM, Warhol MJ, Rando RF, Sedlacek TV, Kemp JE, Fisher JC. Studies on the transmission of viral disease via the CO2 laser plume and ejecta. J Reprod Med. 1990;35:1117-23.

40. Capizzi PJ, Clay RP, Battey MJ. Microbiologic activity in laser resurfacing plume and debris. Lasers Surg Med. 1998;23:172-4.

41. Englehardt RK, Nowak BM, Seger MV, Duperier FD. Contamination resulting from aerosolized fluid during laparoscopic surgery. J Soc Laparoendosc Surg. 2014;18:e2014.00361.

42. Rioux M, Garland A, Webster D, Reardon E. HPV positive tonsillar cancer in two laser surgeons: case reports. J Otolaryngol Head Neck Surg. 2013;42:54.

43. van Doremalen N, Bushmaker T, Morris DH, Holbrook MG, Gamble A, Williamson BN, et al. Aerosol and surface stability of SARS-CoV-2 as compared with SARS-CoV-1. N Engl J Med. 2020;382:1564-7.

44. Zheng MH, Boni L, Fingerhut A. Minimally invasive surgery and the novel coronavirus outbreak: lessons learned in China and Italy. Ann Surgery.2020;272:e5-e6. https://doi.org/10.1097/SLA. 0000000000003924.

45. CAGS issues recommendations to reduce the risk of aerosolization during laparoscopy. Canadian Journal of Surgery, 2020. https://www.facs.org/covid-19/clinical-guidance/additionalclinical.

46. Roach M III. A phase III trial comparing whole pelvic irradiation followed by a conedown boost to boost irradiation only and comparing neoadjuvant to adjuvant total androgen suppression (TAS). https://www.rtog.org/ClinicalTrials/ProtocolTable/StudyDetails.aspx? study $=9413$.

47. Guan WJ, Ni ZY, Hu Y, Liang WH, Ou CQ, He JX, et al. Clinical characteristics of coronavirus disease 2019 in China. N Engl J Med. 2020;382:1708-20.

48. Zhou F, Yu T, Du R, Fan G, Liu Y, Liu Z, et al. Clinical course and risk factors for mortality of adult inpatients with COVID-19 in Wuhan, China: a retrospective cohort study. Lancet. 2020;395:1054-62.

49. Montopoli M, Zumerle S, Vettor R, Rugge M, Zorzi M, Catapano CV, et al. Androgen-deprivation therapies for prostate cancer and risk of infection by SARS-CoV-2: a populationbased study $(\mathrm{n}=4532)$. Annal Oncol. 2020;S0923-7534(20) 39797-0. https://doi.org/10.1016/j.annonc.2020.04.479. [Online ahead of print]. 